\title{
Подвижность и квантовое время бесщелевых дираковских электронов в квантовых ямах НgTe
}

\author{
Институт ффизики полупроводников им. А.В. Ржанова \\ Сибирского отделения Российской академии наук, \\ 630090 Новосибирск, Россия \\ Новосибирский государственный университет, \\ 630090 Новосибирск, Россия \\ E-mail: DobretsovaAA@gmail.com
}

() А.А. Добрецова, З.Д. Квон, Л.С. Брагинский, М.В. Энтин, Н.Н. Михайлов

(Получена 25 апреля 2018 г. Принята к печати 7 мая 2018 г.)

\begin{abstract}
Проведено экспериментальное и теоретическое исследование подвижности и квантового времени дираковских электронов в квантовых ямах HgТе толщиной, близкой к критической, соответствующей переходу от прямого спектра к инвертированному. Экспериментально обнаружена немонотонная зависимость подвижности от электронной концентрации. Построена теория рассеяния дираковских электронов на примесях и неровностях границ ямы, приводящих к флуктуациям толщины ямы. Сравнение указанной теории с экспериментом дает хорошее согласие и объясняет обнаруженную немонотонность уменьшением отношения длины волны де Бройля дираковских электронов к характерному размеру неровностей с ростом электронной концентрации. Установлено, что транспортное время почти на порядок больше квантового, когда доминирует рассеяние на шероховатостях. Также изучен переход от макроскопических образцов к мезоскопическим и обнаружено резкое уменьшение и подвижности, и квантового времени. Предположено, что оно вызвано размерным эффектом на длине свободного пробега.
\end{abstract}

DOI: $10.21883 /$ FTP.2018.11.46598.20

В недавних работах [1-4] было показано, что в квантовых ямах на основе теллурида ртути, ширина которых близка к критической $\left(d_{c}\right)$, соответствующей переходу от прямого спектра к инвертированному, реализуется система однодолинных двумерных дираковских фермионов, имеющих бесщелевую природу и соответственно линейный энергетический спектр (двумерный вейлевский полуметалл), что приводит к целому ряду особенностей в ее транспортном $[2,3]$ и оптическом $[1,4]$ отклике. Однако до настоящего времени отсутствуют детальные и систематические исследования подвижности и квантового времени в указанной системе и информация ограничивается только обрывочными сведениями в работах [2,5]. Данная работа восполняет указанный пробел. В ней проведено исследование поведения подвижности и квантового времени справа от дираковской точки. Обнаружена немонотонная зависимость подвижности от концентрации двумерных электронов, вызванная особенностью их рассеяния на флуктуациях ширины квантовой ямы. Также установлено, что транспортное время заметно превышает квантовое.

Исследованные образцы представляли собой полевые холловские структуры макроскопических и мезоскопических размеров, изготовленные на основе квантовых ям $\operatorname{HgTe}$ с заданной толщиной: 6.3, 6.4, 6.6, 7 нм и ориентацией (013). Стоит отметить, что в отличие от остальных ям, яма толщиной 7 нм соответствует уже состоянию двумерного топологического изолятора. Макроскопические образцы имели ширину 50 мкм и расстояние между потенциометрическими контактами 100 и 250 мкм, а мезоскопические - 3.2 и 35 мкм.
Измерения проводились при температуре $4.2 \mathrm{~K}$ и в магнитных полях до 2 Тл с использованием стандартной схемы фазочувствительного детектирования на частотах 12 Гц и при величинах измерительного тока через образец 100 нА, исключающих эффекты разогрева.

Типичные зависимости проводимости образцов от эффективного затворного напряжения $\sigma\left(V_{g}^{\mathrm{eff}}\right)$ (где $V_{g}^{\text {eff }}=V_{g}-V_{g}^{\mathrm{min}}, V_{g}$ - приложенное затворное напряжение, $V_{g}^{\min }$ - затворное напряжение, соответствующее минимальному значению проводимости) для всех четыpex толщин квантовых ям показаны на рис. 1. Хорошо видно, что все зависимости проходят через минимум, причем минимальное значение проводимости для всех кривых превышает $e^{2} / h$, что подтверждает сделанный в [2] вывод о квазиметаллическом поведении ям с толщиной, близкой к критической, в дираковской точке. Также отметим, что в окрестности дираковской точки они имеют вид, близкий к симметричному относительно $V_{g}^{\text {eff }}=0$. При дальнейшем росте амплитуды затворного напряжения указанная симметрия теряется из-за близости долин тяжелых дырок [2]. Далее в работе будет рассматриваться только поведение проводимости справа от дираковской точки, когда перенос осуществляется дираковскими электронами (ДЭ). Анализ поведения дираковских дырок осложнен влиянием уже упомянутых боковых долин тяжелых дырок $[2,6]$ и выходит за рамки данной работы.

На рис. 2 показаны зависимости подвижности электронов $\mu$, определенные из проводимости, от концентрации $N_{s}$ для образцов макроразмера. Видно, что подвижность ям толщиной 6.3 и $6.4 \mathrm{Hм}$ растет во 


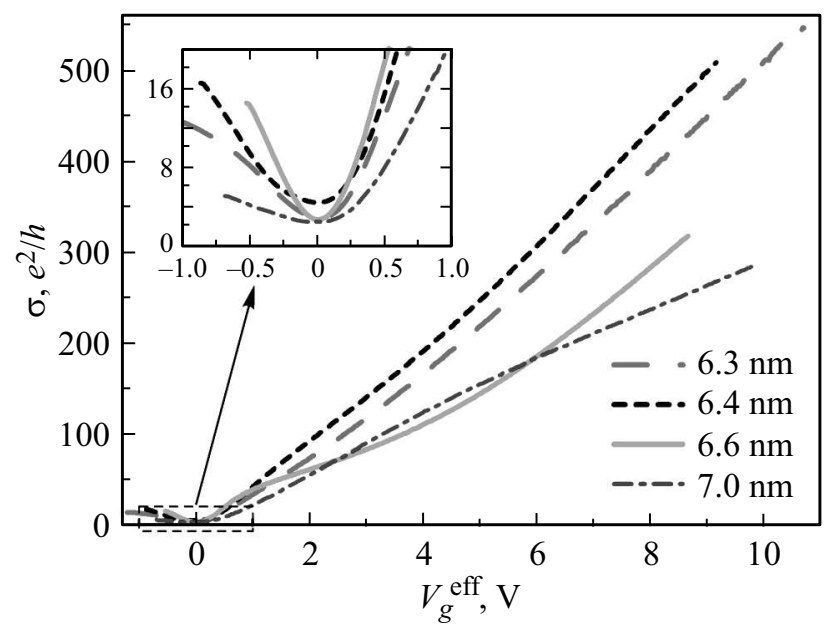

Рис. 1. Типичные зависимости проводимости безмассовых дираковских фермионов в зависимости от эффективного затворного напряжения $V_{g}^{\text {eff }}=V_{g}-V_{g}^{\min }$ при температуре $T=4.2 \mathrm{~K}$.

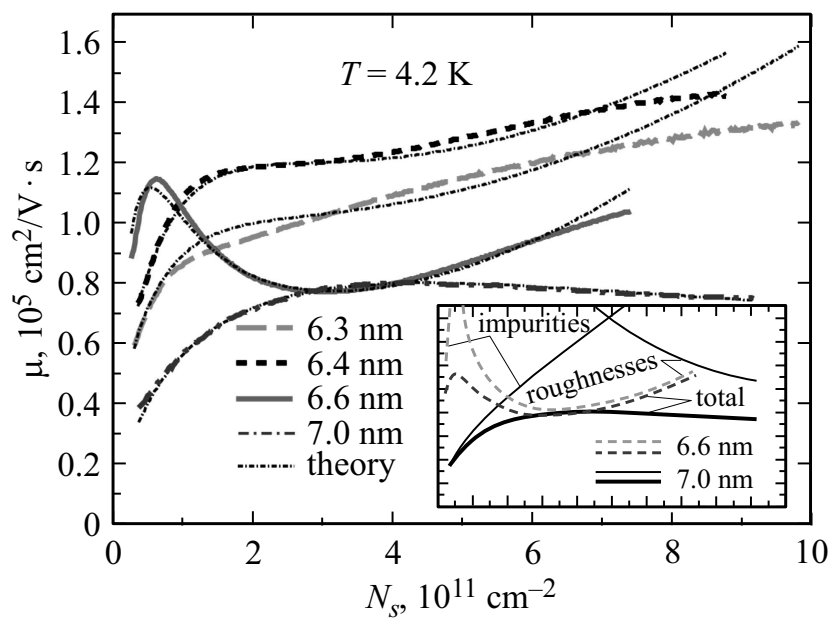

Рис. 2. Подвижность электронов в макроскопических образцах с одиночной квантовой ямой $\mathrm{HgTe}$ толщиной 6.3, 6.4, 6.6 и 7 нм в зависимости от концентрации. Тонкие линии соответствуют теоретическому расчету. На вставке - вклады рассеяния на примесях и шероховатостях в расчетные кривые для 6.6 и 7 нм ям.

всем диапазоне концентраций, при этом при концентрации $N_{s}=(0.9-1.3) \cdot 10^{11} \mathrm{~cm}^{-2}$ наблюдается изменение скорости роста подвижности с более резкого при малых концентрациях на более плавный при больших концентрациях. Также для ям $6.4 \mathrm{Hм}$ наблюдается перегиб в подвижности при концентрации $N_{s} \approx 2.8 \cdot 10^{11} \mathrm{~cm}^{-2}$. Другое поведение наблюдается для ям толщиной 6.6 нм, где при малых концентрациях, как и в случае ям толщиной 6.3 и 6.4 нм, подвижность резко растет, затем, однако, при концентрации электронов $N_{s}=(0.5-1) \cdot 10^{11} \mathrm{~cm}^{-2}$ она достигает максимума, равного $(1-2) \cdot 10^{5} \mathrm{~cm}^{2} / \mathrm{B} \cdot \mathrm{c}$ в зависимости от конкретного образца, затем начинает падать, проходит через заметно более пологий, чем максимум, минимум при
$N_{s} \approx 3 \cdot 10^{11} \mathrm{~cm}^{-2}$ и, наконец, после этого снова начинает расти, но уже значительно медленнее. В случае ям толщиной 7 нм подвижность так же, как и в предыдущих случаях, растет при малых концентрациях электронов и затем, начиная с концентрации $N_{s} \approx 4 \cdot 10^{11} \mathrm{~cm}^{-2}$, плавно уменьшается. Поведение подвижности, наблюдаемое для ям толщиной 7 нм, является аналогичным тому, что наблюдается в других структурах: кремниевых инверсионных каналах [7], GaAs-гетероструктурах [8], широких квантовых ямах $\mathrm{HgTe}$ [5,9]. Немонотонное же поведение подвижности, полученное для ям толщиной $6.6 \mathrm{Hм}$, не наблюдалось ранее ни в одной из перечисленных систем.

Для проведения анализа указанного аномального поведения подвижности в работе была построена теория рассеяния дираковских электронов на удаленных примесях и флуктуациях толщины квантовой ямы. При этом, так как температура в эксперименте была значительно ниже энергии Ферми $\left(E_{f}>20\right.$ мэВ), температура в теории полагалась равной нулю. Остановимся кратко на основных моментах теории. Очевидно, что стандартный подход к примесному рассеянию, давно известный для двумерных электронов с параболическим спектром (см., например, $[7,10])$, в данном случае не годится и необходимо учесть линейность спектра дираковских электронов, что нетрудно сделать, модифицировав теорию для массивных двумерных электронов. Тогда выражение для транспортного времени релаксации будет иметь следующий вид:

$$
\begin{aligned}
\frac{1}{\tau_{\text {imp }}}= & N_{\text {imp }} \frac{8 \pi e^{4}}{\kappa^{2} h^{3} s^{2}} E_{f} \int_{0}^{\pi} \frac{\sin ^{2} \varphi}{\left(2 k_{f} \sin \varphi+q_{0}\right)^{2}} \\
& \times\left(\cos ^{2} \varphi+\frac{\delta^{2}}{E_{f}^{2}} \sin ^{2} \varphi\right) \exp \left(-4 k_{f} d_{s p} \sin \varphi\right) d \varphi
\end{aligned}
$$

где $N_{\text {imp }}-$ концентрация примесей, $E_{f}=$ $=\sqrt{\delta^{2}+\hbar^{2} s^{2} k_{f}^{2}}-$ уровень Ферми дираковских электронов, $k_{f}-$ волновой вектор Ферми, $s \approx 7 \cdot 10^{7} \mathrm{~cm} / \mathrm{c}[11]$ - скорость Ферми, $\delta$ - щель в спектре, $d_{s p}$ - расстояние от примесей до электронов, $q_{0}=2 e^{2} E_{f} / \kappa \hbar^{2} s^{2}-$ волновой вектор Томаса-Ферми для ДЭ, $\kappa-$ диэлектрическая проницаемость квантовой ямы HgTe.

Рассмотрим теперь рассеяние, вызванное неровностями квантовой ямы. Теория данного рассеяния для ям с параболическим спектром была построена почти полвека назад в [12] и давно является канонической [7]. Однако в нашем случае ее необходимо модифицировать, так как в ямах с критической толщиной ее флуктуации могут привести к флуктуациям щели в спектре носителей заряда, которые и приводят к релаксации импульса. Учесть данные флуктуации можно, заложив в гамильтониан Дирака, описывающий ДЭ, зависимость щели от толщины ямы, что в предположении малости флуктуаций 
толщины с точностью до первого порядка дает

$$
\begin{aligned}
H(\mathbf{k}) & =\hbar s \boldsymbol{\sigma} \mathbf{k}+\left[\delta+\left(\frac{\partial \delta}{\partial d}\right)_{d=d_{0}} \xi(\boldsymbol{\rho})\right] \sigma_{z} \\
& =H_{0}(\mathbf{k})+\left(\frac{\partial \delta}{\partial d}\right)_{d=d_{0}} \xi(\boldsymbol{\rho}) \sigma_{z},
\end{aligned}
$$

где $H_{0}(\mathbf{k})-$ невозмущенный гамильтониан, $\mathbf{k}=\left(k_{x}, k_{y}\right)-$ волновой вектор в плоскости ямы, $\sigma_{i}$ - матрицы Паули, $\xi(\boldsymbol{\rho})=\left[d(\boldsymbol{\rho})-d_{0}\right]-$ флуктуация толщины ямы, $d_{0}-$ средняя толщина ямы.

Тогда по теории возмущения можно получить следующее транспортное время рассеяния на флуктуациях щели:

$$
\begin{aligned}
\frac{1}{\tau_{r}} & =\frac{2 H^{2} L^{2}}{\hbar^{3} s^{2}}\left(\frac{\partial \delta}{\partial d}\right)^{2} E_{f} \\
& \times \int_{0}^{\pi} \sin ^{2} \varphi\left(\frac{\delta^{2}}{E_{f}^{2}} \cos ^{2} \varphi+\sin ^{2} \varphi\right) \exp \left[-k_{f}^{2} L^{2} \sin ^{2} \varphi\right] d \varphi .
\end{aligned}
$$

При выводе данного выражения было предположено, что корреляционная функция шероховатостей имеет гауссов вид

$$
W(\rho)\left\langle\xi(\boldsymbol{\rho}) \xi\left(\boldsymbol{\rho}^{\prime}+\boldsymbol{\rho}\right)\right\rangle=H^{2} \exp \left[-\rho^{2} / L^{2}\right],
$$

где $H$ и $L-$ параметры, которые описывают характерные высоту и длину неровностей соответственно. Зная транспортное время рассеяния, можно вычислить подвижность, выражение для которой в случае линейного спектра также будет отличаться от выражения в случае параболических электронов:

$$
\mu=-\frac{e}{2} \frac{\int_{0}^{\infty} \tau(E) v(E)^{2} N(E) f_{0}^{\prime} d E}{\int_{0}^{\infty} N(E) f_{0}^{\prime} d E}=\frac{e \tau\left(E_{f}\right) s^{2}}{E_{f}},
$$

где $N(E)=E / \pi \hbar^{2} s^{2}-$ плотность состояний ДЭ, $f_{0}-$ функция распределения Ферми-Дирака.

Перейдем теперь к сравнению экспериментальных данных с теоретическим расчетом. Сделаем несколько предварительных замечаний. При расчете подвижности, обусловленной примесным рассеянием, предполагалось, что в исследованных структурах оно вызвано прежде всего примесями легирующих слоев, расположенных на расстоянии $\sim 20 \mathrm{Hм}$ от границ ямы и имеющих концентрацию $(7.5-9.5) \cdot 10^{10} \mathrm{~cm}^{-2}$, а также неоднородно распределенными вблизи границы $\mathrm{HgTe} / \mathrm{HgCdTe}$ остаточными примесями. В расчете они моделировались заряженной плоскостью, расположенной на некотором расстоянии от ямы. Соответственно вводились два подгоночных параметра: концентрация примесей $N_{\text {imp }}$ и их среднее расстояние до максимума волновой функции электрона $d_{s p}$.
Параметры ям, полученные при подгонке теоретических кривых к экспериментальным

\begin{tabular}{c|c|c|c|c|c}
\hline \multicolumn{1}{c|}{$d, \mathrm{HM}$} & $\delta$, мэВ & $N_{\text {imp }}, 10^{11} \mathrm{~cm}^{-2}$ & $d_{s p}, \mathrm{HM}$ & $L, \mathrm{HM}$ & $H, \mathrm{HM}$ \\
\hline 6.3 & 5 & 4.5 & 10 & 6.5 & 0.18 \\
6.3 (мезо) & 5 & 9.1 & 10 & 6.0 & 0.25 \\
6.4 & 2.5 & 5.3 & 13 & 6.3 & 0.17 \\
6.6 & -2.5 & 5.9 & 25 & 7.8 & 0.24 \\
7.0 & -12.5 & 4.0 & 5 & 3.0 & 0.25
\end{tabular}

Примечание. $\delta$ - энергетическая щель в спектре дираковских фермионов, $N_{\text {imp }}$ - концентрация примесей, $d_{s p}$ - среднее расстояние примесей до максимума волновой функции электрона, $L$ и $H-$ характерные длина и высота шероховатостей (при этом предполагалось, что

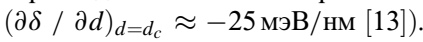

Результаты сравнения теории с экспериментом показаны на рис. 2. Хорошо видно, что расчет удовлетворительно описывает все экспериментальные зависимости $\mu\left(N_{s}\right)$. Параметры, полученные при подгонке теоретических кривых к экспериментальным, приведены в таблице. Значения щели в спектре дираковских электронов были взяты из [13] с поправкой на то, что из-за напряжения ямы, вызванного различием постоянных решетки $\mathrm{HgTe}$ и $\mathrm{CdTe}$, критическая толщина ямы равна 6.5 нм [14]. Обсудим величины полученных подгоночных параметров и их поведение, а затем отдельно наиболее важный вопрос: как предложенная теория объясняет немонотонное поведение подвижности. Если говорить о параметрах примесного рассеяния, то они показывают, что рассеивающие примеси сосредоточены в основном вблизи областей легирования, однако их концентрация в несколько раз превышает заданную. Как и в других двумерных системах, рассеяние на заряженных примесях доминирует при малых $N_{s}$. При дальнейшем увеличении $N_{s}$ начинает превалировать уже рассеяние на неровностях, приводя к тому, что в случае ям толщиной 6.3 и 6.4 нм рост подвижности резко ослабляется, а в случае ямы 7 нм он сменяется небольшим падением. В случае же 6.6 нм ямы, как было отмечено ранее, рост подвижности сменяется падением, но затем снова сменяется ростом. Такое поведение наблюдалось впервые и кажется на первый взгляд неожиданным. Скорее всего, это связано с тем, что в эксперименте ни в одной из ранее исследованных систем указанный рост подвижности не наблюдался. Однако в теории рассеяния на шероховатостях тех же параболических электронов такой рост уже содержится и связан он с тем, что с увеличением $N_{s}$ уменьшается длина волны де Бройля электрона $\lambda_{e}$ и возможен переход от условия $k_{f} L<1$ к обратному $k_{f} L>1$, сопровождаемый сменой зависимости $\mu\left(N_{s}\right)$ от уменьшения $\mu$ к ее увеличению. Более того, на возможность наблюдения такого перехода в квантовых ямах $\mathrm{Si} / \mathrm{SiGe}$ было специально обращено внимание в работе [15]. Однако концентрация, необходимая для этого, превышала $3 \cdot 10^{12} \mathrm{~cm}^{-2}$, а наиболее высокая концентрация электронов, достигнутая в таких ямах, равна 


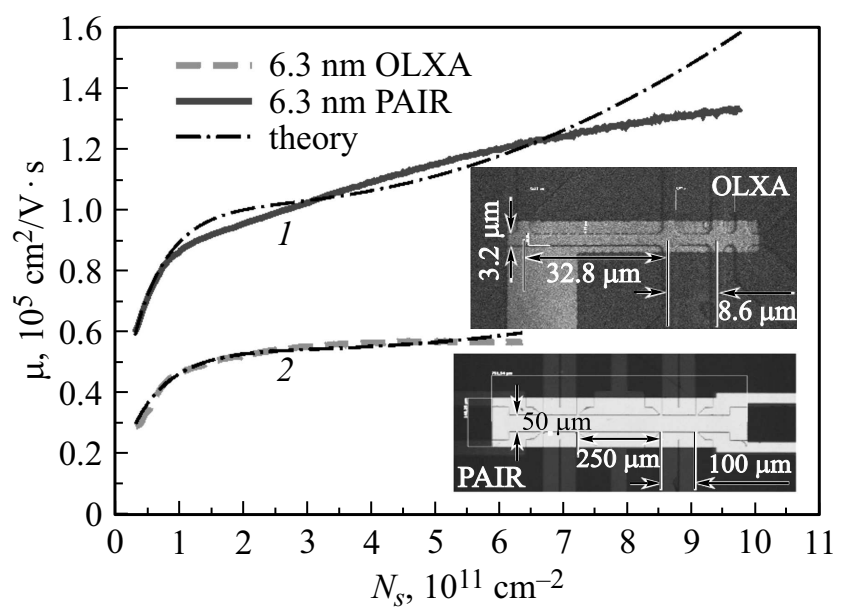

Рис. 3. Подвижность электронов для макро- (1) и мезоскопического (2) образцов с квантовой ямой $\mathrm{HgTe}$ толщиной $6.3 \mathrm{Hм}$ в зависимости от концентрации. На вставках - фотографии мезо- и макроскопического образцов, исследуемых в работе.

$10^{12} \mathrm{~cm}^{-2}$. Так или иначе, но, по-видимому, в данной работе немонотонная зависимость $\mu\left(N_{s}\right)$, обусловленная рассеянием на неровностях, обнаружена впервые.

Для ям толщиной 6.4 и $6.3 \mathrm{Hм}$, как отмечено ранее, хорошо выраженный минимум подвижности не наблюдается, и вместо него возникает точка перегиба. Наличие минимума в зависимости суммарной подвижности от концентрации определяется двумя факторами: относительными величинами примесной подвижности и подвижности при рассеянии на шероховатостях, а также характерной длиной шероховатостей $L$, которая определяет концентрацию, соответствующую началу роста подвижности при рассеянии на шероховатостях. Таким образом, по-видимому, в силу того что в ямах толщиной 6.3 и 6.4 нм примесная подвижность заметно меньше, чем в ямах толщиной 6.6 нм, минимум подвижности в них не возникает, хотя, как следует из таблицы, параметры шероховатостей для ямы 6.6 нм практически не отличаются от таковых для ям 6.3 и 6.4 нм. Примесная подвижность мала также и в ямах толщиной 7 нм. Однако, так как для них характерная длина шероховатостей получилась существенно меньшей, то начиная с $N_{s} \approx 4 \cdot 10^{11} \mathrm{~cm}^{-2}$ в них наблюдается падение подвижности, а не рост, как в случае ям толщиной 6.3 и 6.4 нм. Также заметим, что данные о поведении $\mu\left(N_{s}\right)$ в аналогичном диапазоне $N_{s}$ были приведены в [5] для

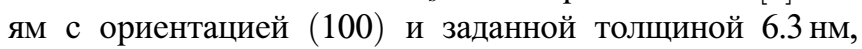
при этом указанная зависимость не содержала никаких аномалий. Это позволяет предположить, что характер неровностей на границе квантовой ямы HgTe зависит от ее ориентации, точнее характерная длина неровностей для ям с ориентацией (100), по-видимому, существенно меньше, чем для ям с ориентацией (013), и условие $k_{f} L<1$ соблюдается в них во всем исследуемом диапазоне $N_{s}$.
В работе на основе анализа осцилляций Шубниковаде Гааза было также определено квантовое время для электронных концентраций, когда доминирует рассеяние на шероховатостях. При $N_{s} \approx 5 \cdot 10^{11} \mathrm{~cm}^{-2}$ оно равно $10^{-13}$ c, т.е. почти на порядок меньше транспортного времени. Подобный результат снова ставит вопрос о том, с чем связано такое разногласие, так как рассеяние на шероховатостях является короткодействующим и полученную разность времен нельзя объяснить разным механизмом транспортного и квантового времен, как в двумерном электронном газе на основе гетероструктур и ям $\mathrm{AlGaAs} / \mathrm{GaAs}$.

В заключительной части работы вышеописанные измерения были проведены для образцов с узкими каналами (3.2 мкм), созданными на основе квантовой ямы толщиной 6.3 нм. Было обнаружено (рис. 3) заметное, почти в 2 раза, падение подвижности в сравнении с макроскопическими образцами, изготовленными на основе той же ямы. Более того, квантовое время уменьшилось еще сильнее. Возможно, указанные результаты свидетельствуют о более сильном проявлении размерных эффектов на длине свободного пробега в системе бесщелевых дираковских фермионов, чем для параболических электронов. Однако для более определенных выводов требуются дальнейшие более детальные исследования.

Работа выполнена при поддержке РФФИ (грант № 17-52-14007).

\section{Список литературы}

[1] З.Д. Квон, С.Н. Данилов, Д.А. Козлов, К. Цот, Н.Н. Михайлов, С.А. Дворецкий, С.Д. Ганичев. Письма ЖЭТФ, 94, 895 (2011).

[2] Д.А. Козлов, З.Д. Квон, Н.Н. Михайлов, С.А. Дворецкий. Письма ЖЭТФ, 96, 815 (2012).

[3] Д.А. Козлов, З.Д. Квон, Н.Н. Михайлов, С.А. Дворецкий. Письма ЖЭТФ, 100, 824 (2014).

[4] C. Zoth, P. Olbrich, P. Vierling, K.-M. Dantscher, V.V. Bel'kov, M.M. Glazov, L.E. Golub, D.A. Kozlov, Z.D. Kvon, N.N. Mikhailov, S.A. Dvoretsky, S.D. Ganichev. Phys. Rev. B, 90, 205415 (2014).

[5] G. Tkachev, C. Thienel, B. Büttner, C. Brüne, H. Buhmann, L.W. Molenkamp, E.M. Hankiewicz. Phys. Rev. Lett., 106, 076802 (2011).

[6] B. Büttner, C.X. Liu, G. Tkachov, E.G. Novik, C. Brüne, H. Buhmann, E.M. Hankiewicz, P. Recher, B. Trauzettel, S.C. Zhang, L.W. Molenkamp. Nature Phys., 7, 418 (2011).

[7] T. Ando, A. Fowler, F. Stern. Rev. Mod. Phys., 54, 437 (1982).

[8] H. Sakaki, T. Noda, K. Hirakawa, M. Tanaka, T. Matsusue. Appl. Phys. Lett., 51, 1934 (1987).

[9] А.А. Добрецова, Л.С. Брагинский, М.В. Энтин, З.Д. Квон, Н.Н. Михайлов, С.А. Дворецкий. Письма ЖЭТФ, 101, 360 (2015).

[10] J.H. Davies. The Physics of Low-dimensional Semiconductors (Cambridge University Press, 1998). 
[11] P. Olbrich, C. Zoth, P. Vierling, K.-M. Dantscher, G.V. Budkin, S.A. Tarasenko, V.V. Belkov, D.A. Kozlov, Z.D. Kvon, N.N. Mikhailov, S.A. Dvoretsky, S.D. Ganichev. Phys. Rev. B, 87, 235439 (2013).

[12] A.V. Chaplik, M.V. Entin. Soviet Phys. JETP, 28, 514 (1968)

[Zh. Eksp. Teor. Fiz., 55, 990 (1968)].

[13] O.E. Raichev. Phys. Rev. B, 85, 045310 (2012).

[14] Е.Л. Новик, частное сообщение.

[15] A. Gold. Phys. Rev. B, 35, 723 (1987).

Редактор Л.В. Шаронова

\title{
Gapless Dirac electrons mobility and quantum time in HgTe quantum wells
}

\author{
A.A. Dobretsova, Z.D. Kvon, L.S. Braginskii, \\ M.V. Entin, N.N. Mikhailov
}

Rzhanov Institute of Semiconductor Physics, Siberian Branch of Russian Academy of Sciences, 630090 Novosibirsk, Russia

Novosibirsk State University, 630090 Novosibirsk, Russia

\begin{abstract}
Dirac electrons mobility and quantum time in quantum wells based on HgTe with width close to critical, corresponding to the transition from normal to inverted spectrum, have been investigated experimentally and theoretically. Non-monotonic mobility dependence on electron concentration has been observed experimentally. The theory of Dirac electrons scattering on impurities and quantum well width fluctuations due to heterointerfaces roughnesses has been developed. The theory fits well the experiment and explains non-monotonic mobility behavior by decreasing the ratio of Dirac electrons de Broglie wave length to the roughness typical size with the electron concentration increase. Quantum time has been found to be almost one order smaller than the transport time when roughness scattering is dominating. Also the transition from macro- to mesoscopic samples has been studied, abrupt mobility and quantum time decrease has been observed. The decrease was proposed to be induced by size effect at the mean free pass length.
\end{abstract}

